\title{
Optimal Link Capacity Dimensioning in Proportionally Fair Networks
}

\author{
Michał Pióro ${ }^{1}$, Gábor Malicskó ${ }^{2}$, and Gábor Fodor ${ }^{3}$ \\ 1 Department of Communication Systems, Lund Institute of Technology, Sweden, \\ Michal.Pioro@telecom.1th.se \\ 2 Ericsson Traffic- and Performance Laboratory, Hungary, \\ Gabor.Malicsko@eth.ericsson.se \\ 3 Ericsson Research, Sweden, Gabor.Fodor@era-t.ericsson.se
}

\begin{abstract}
We consider the problem of link capacity dimensioning and bandwidth allocation in networks that support elastic flows and maintain proportional fairness among these flows. We assume that a certain allocated bandwidth to a user demand generates revenue for the network operator. On the other hand, the operator is incurred a capacity dependent cost for each link in the network. The operator's profit is the difference between the revenue and the total link cost. Under this assumption the problem is to determine the bandwidth of the flows and the link capacities such that the profit is maximized. We first show that under fairly general assumptions, the optimum allocation of flows leads to selecting the lowest cost paths between O-D pairs. We also derive explicit formulae for the bandwidth allocated to these flows. We distinguish the case when the operator's capacity budget is fixed ("equality budget constraint", in which case the profit is maximized when the revenue is maximized) and the case when the budget is upper-bounded ("inequality budget constraint", in which case the profit can - in general - be maximized by using some portion of the capacity budget). Finally, we show numerical examples to highlight some of the trade-offs between profit maximization, revenue maximization and fairness.
\end{abstract}

Keywords: network dimensioning, bandwidth allocation, routing, traffic engineering, linear programming, convex optimization

\section{Introduction}

After years of research and standardization efforts, there seems to be a growing consensus that some form of traffic engineering and in particular the separation of flows with different quality of service (QoS) demands are necessary to avoid too costly over-dimensioning of IP networks [1], [2]. To this end, MPLS provides a set of standards that can be applied to explicitly allocate bandwidth resources between originator-destination (O-D) pairs [13]. In addition, traffic engineering algorithms can also be useful to provide some kind of a "reference engineered network" that can help operators to determine the level of over-dimensioning in a non-engineered network. Despite these obvious motivations, it is however still the topic of research and standardization exactly which mechanisms and algorithms can be used in for instance MPLS networks for various aspects of traffic engineering. 
Despite numerous recent advances (see Section 2 for a more detailed discussion), adopting the "traditional" traffic engineering methods (including link capacity dimensioning, routing and bandwidth allocation) from circuit switched networks (such as PSTN's, ISDN's or even ATM networks) is non-trivial, because of the presence of elastic traffic classes.

Therefore, in this paper we concentrate on developing a model and algorithms that take into account the above three aspects of engineering for elastic services. This paper builds upon the results of the first author in [17] where the detailed proofs of the results are presented. Specifically, we assume that between each O-D pair, there may be only a single user flow realizing the demand, i.e. we exclude the case of the "demand split".

Under this assumption, the network operator faces the following problems:

- The capacity of each link must be determined such that the network can accommodate the offered traffic (dimensioning).

- The traffic demand between the O-D pair must be associated either with a single flow or - in the case when demand split is acceptable - with a set of flows, and an appropriate route must be found for each flow (routing).

- Bandwidth must be allocated for each flow such that some notion of fairness among the user flows is maintained (bandwidth allocation).

In our model, each user flow generates a bandwidth dependent revenue for the operator. On the other hand, each link incurs a capacity dependent cost for the operator, who is therefore motivated in maximizing the resource utilization in the network (and thereby its profit which is defined as the difference between revenue and cost). The network operator may be interested in maximizing its profit while keeping the total link cost fixed ("equality link cost budget constraint") or he may want to maximize the profit while keeping the total link cost under some bound ("inequality budget constraint"). Note that in the first case the profit is maximized when the revenue is maximized.

While there is no "killer argument" or any clear technical or economical evidence why a certain (if any) fairness between user flows should be maintained, maximizing the network throughput may lead to extremely unfair allocations (including the situation where some flows are deemed to complete starvation), see for instance [16].

Therefore, we will formulate the traffic engineering problem as a series of optimization tasks, where one is interested in maximizing the profit/revenue subject to capacity and fairness constraints. Specifically, we will consider the following two cases:

1. Dimensioning under a fixed budget constraint, the routes are considered fixed and pre-determined to each flow. (Task 1)

2. Profit (revenue-cost) maximization, where the budget (i.e. the sum of all link costs) is not fixed but can be freely chosen up to a limit. (Task 2)

Throughout the numerical evaluation of the techniques enumerated above we will use the proportional fair sharing with fixed link capacities and shortest path routing as a reference method (Task 3 ).

We organize the paper as follows. In the next section we take a look at recent research results in the area of bandwidth allocation in (max-min and proportional) fair networks. In Section 3 we formulate the problem of link capacity dimensioning and routing as a 
(revenue) optimization problem under budget and fairness constraints. We assume that the total link cost in the network should be equal to a pre-determined budget ("equality budget constraint"). For this case, we present explicit formulae to determine the allocated bandwidth for each flow. Next, we define the profit optimization task, in which case the sum of all link costs is bounded ("inequality budget constraint"). Here the task is to find both the link cost budget and the bandwidth allocated to flows (and associated revenue) such that the profit is maximized. In Section 6 we discuss numerical results. We conclude in Section 7 .

\section{Related Works}

In the context of routing and resource allocation under fairness constraints, most paper consider the popular max-min fairness mostly in Asynchronous Transfer Mode (ATM) Available Bit Rate (ABR) context, since the ATM Forum adopted the max-min fairness criterion to allocate network bandwidth for ABR connections [7], [19], [21]. However, these papers do not consider the issue of path optimization in the bounded elastic environment. For instance, [21] studies the speed of convergence of max-min fair allocation algorithms rather than focusing on path or link capacity optimization.

From the point of view whether the flows are static (also called "long lived") or dynamic (where some arrival and departure patterns are also considered) these papers can be divided into two groups. Representative papers for the static case include for instance the papers by Kleinberg et al., see [11] and [12]. Another important series of work on the static case is the papers that develop on-line fair routing algorithms where the demand matrix is not a-priori known, see for instance [6], [18] and more recently [8].

The "dynamic case" under both max-min and proportional fairness is analyzed, mostly focusing on stability aspects, by Veciana et al. in [22]. Here, routes and link capacities are assumed fixed.

Max-min fair routing is the topic of the paper by Ma et al. [14], where the widestshortest, shortest-widest and the shortest-dist algorithms are studied. These algorithms do not aim to explicitly maximize the carried traffic and consequently the path allocation is not formulated as an optimization task.

An important reference for both the static- and dynamic cases is the series of works by Massoulie and Roberts, see e.g. [15] and [16]. Here, a number of fairness notions are discussed and associated optimization tasks are presented for the case of the unbounded flows and assuming fixed routes and link capacities.

Although the max-min allocation has been widely accepted and studied in the literature, its appropriateness can be questioned because of the relatively low bandwidth utilization. One of the promising alternatives to the max-min fairness is the proportional-rate fairness proposed by Kelly in [9], [10] and also summarized by Massoulie and Roberts in [16]. Because of its superior characteristics in terms of overall network utilization, we in this paper concentrate on the proportional fair allocation method.

According to the proportional-rate fairness criterion, the rate allocations $x_{d}$ are fair, if they maximize $\sum_{d} \log x_{d}$ (or in the weighted case $\sum_{d} w_{d} \log x_{d}$, where $w_{d}$ is the weight of demand $d$ ) under the capacity constraints. This objective may be interpreted as being 
to maximize the overall revenue of allocations assuming each route has a logarithmic revenue function.

Bandwidth allocation algorithms can further be divided into two main groups. The ERAQLES algorithm in ATM [3] or the algorithms of [16] provide examples on distributed algorithms, while the application of a bandwidth broker facilitates the use of centralized bandwidth allocation algorithms [20].

In an earlier work [5], we developed centralized algorithms for networks with given (fixed) link capacities that optimize routing in order to improve the available level of fairness (either max-min or proportional rate) between flows. In [4] we combined network dimensioning and proportional fair bandwidth allocation into a single optimization task without the inequality budget constraint.

Our contribution to this line of works is the development of explicit analytical formulae for a set of optimization tasks that allow for the joint optimization of routing and link capacity dimensioning. To the best of our knowledge, such a profit/revenue optimization formulation and associated formulae and algorithms have not yet been proposed in the literature.

\section{Fixed Budget Network Dimensioning for Non-bounded Elastic Flows}

As a basic case, consider the following optimization task, where the objective is to find the rate allocations $x_{d}$ and link capacities $y_{e}$ such that the logarithmic revenue function corresponding to proportional fairness is maximized. Note that each link $e$ is associated with a marginal cost $c_{e}$ and so the operator's total link cost is $\sum c_{e} y_{e}$. In this basic setting we assume that the operator's total link cost budget $(C)$ is kept fixed such that $C=\sum c_{e} y_{e}$.

Task 1. Dimensioning under Fixed (Equality) Budget Constraint with Fixed Routes indices:

$$
\begin{aligned}
& d=1,2, \ldots, D \text { demands } \\
& e=1,2, \ldots, E \text { links }
\end{aligned}
$$

\section{constants:}

$$
a_{e d}=\left\{\begin{array}{l}
1 \text { if link } e \text { belongs to the path realizing demand } d \\
0 \text { otherwise. }
\end{array}\right.
$$

$w_{d}$ weight of demand $d$

$c_{e}$ marginal cost of link $e$

$C$ assumed budget

\section{variables:}

$x_{d}$ flow allocated to demand $d$

$y_{e}$ capacity of link $e$ 
maximize:

$$
F(x)=\sum_{d} w_{d} \log \left(x_{d}\right)
$$

constraints:

$$
\begin{aligned}
\sum_{e} c_{e} y_{e} & =\quad C ; \\
\sum_{d} a_{e d} x_{d} & \leq \quad y_{e} ; \quad e=1,2, \ldots, E
\end{aligned}
$$

$x, y$ non-negative.

The explicit solution of the optimization task is given by the following theorem. Theorem 1. Let $x^{0}$ and $y^{0}$ be the solution of the above task. Then:

$$
\begin{aligned}
\left.F(x)\right|_{x=x^{0}}= & \log (C) \sum_{d} w_{d}-\sum_{d} w_{d} \log \left(\sum_{e} c_{e} a_{e d}\right)+ \\
& +\sum_{d} w_{d} \log \left(w_{d}\right)-\log \left(\sum_{d} w_{d}\right) \sum_{d} w_{d} \\
x_{d}^{0}= & C w_{d} /\left(\left(\sum_{d} w_{d}\right)\left(\sum_{e} c_{e} a_{e d}\right)\right) \\
y_{e}^{0}= & \sum_{d} a_{e d} x_{d}^{0} .
\end{aligned}
$$

Proof: As shown in [17], the dual function for Task 1 is of the form (7) where $\sigma$ is a non-negative dual variable (multiplier) corresponding to constraint (2).

$$
W(\sigma)=\sum_{d}\left(w_{d}-w_{d} \log \left(w_{d} / \sigma \sum_{e} c_{e} a_{e d}\right)\right)-\sigma C .
$$

The maximum of the dual function is attained at the stationary point of (7) with respect to the multiplier :

$$
\sum_{d} w_{d} / \sigma-C=0
$$

Hence, the optimal multiplier $\sigma^{0}$ is given by

$$
\sigma^{0}=\sum_{d} w_{d} / C
$$

and this immediately implies (4) and (5).

Naturally, at the optimum the constraint of inequality (3) is binding. We note that the maximum value of the objective function (1) depends only on $\mathrm{C}$; (1) implies that this maximum is of the form

$$
F(C)=\alpha \log (C)+\beta-\gamma
$$


where $\alpha=\sum_{d} w_{d}, \beta=\sum_{d} w_{d} \log \left(w_{d}\right)-\log \left(\sum_{d} w_{d}\right) \sum_{d} w_{d}$ and $\gamma=\sum_{d} w_{d} \log \left(\sum_{e} c_{e} a_{e d}\right)$.

Formula 10 implies that when the paths for the flows realizing the demands and the link capacities are also subject to optimization then the optimal solution will assign each flow to its shortest (lowest cost with respect to the $c_{e}-\mathrm{s}$ ) path . This is because in (10) only $\gamma$ depends on the path selection and it is minimized when the lowest cost paths are used for realizing the demands' flows.

\section{Profit Maximization}

During the dimensioning of a network the budget constraint usually appears only as an upper limit on the disposable amount of money and the target is to achieve an investment that maximizes the difference of the revenue and the total link costs ("profit"). The revenue associated with demand $d$ depends on the operator's charging model and is not necessarily a linear function of the allocated bandwidth $\left(x_{d}\right)$. In fact, a logarithmic revenue function can be considered appropriate [23]. This logarithmic function is also motivated by the observation that the revenue can become negative if the allocated bandwidth is smaller than a threshold value. The optimization task in accordance with this objective is the following:

Task 2. Profit Maximization under Flexible (Inequality) Budget Constraint with Fixed Routes maximize:

$$
\Psi=\sum_{d} w_{d} \log \left(x_{d}\right)-\sum_{e} c_{e} y_{e}
$$

constraints:

$$
\begin{array}{cl}
\sum_{e} c_{e} y_{e} & \leq C_{0} \\
\sum_{d} a_{e d} x_{d} & \leq y_{e} ; e=1,2, \ldots, E \\
x, y \text { non-negative. } &
\end{array}
$$

The maximal value of the objective function (11) is attained at the maximum, with respect to variable $C$, of the function

$$
\begin{gathered}
F(C) \quad=\quad \log (C) \sum_{d} w_{d}-C \\
\text { over } 0 \leq C \leq C_{0} .
\end{gathered}
$$

The optimum of (14) is attained either at $C=\sum_{d} w_{d}$ (if $\sum_{d} w_{d} \leq C_{0}$ ) or at $C_{0}$ (if $\sum_{d} w_{d}>C_{0}$ ). Of course, the optimal $C$ is the optimal total cost of links $\sum_{e} c_{e} y_{e}$. Furthermore, from (4) it follows that in the case of $\sum_{d} w_{d} \leq C_{0}$ the optimal value of (11) is equal to

$$
\sum_{d} w_{d} \log \left(w_{d} / \xi_{d}\right)-\sum_{d} w_{d}
$$


(where $\xi_{d}$ is the length of the shortest path of demand $d$ ), and the optimal flows are given by

$$
x_{d}^{0}=w_{d} / \xi_{d} ; \quad d=1,2, \ldots, D
$$

\section{The Fixed Link Capacity Method}

We are interested in analyzing the differences between the method outlined in Task 1 and Task 2 and the proportional fair allocation mechanism used for fixed link capacities. In order to do this we need to formulate explicitly the allocation task used for comparisons. The numerical results are presented in Section 6 .

Task 3. Proportional Fair Allocations for Links of Fixed Capacity and Lowest Cost Path Routing indices:

$$
\begin{aligned}
& d=1,2, \ldots, D \text { demands } \\
& e=1,2, \ldots, E \text { links }
\end{aligned}
$$

constants:

$$
a_{e d}=\left\{\begin{array}{l}
1 \text { if link } e \text { belongs to the path realizing demand } d \\
0 \text { otherwise }
\end{array}\right.
$$

$\begin{array}{ll}w_{d} & \text { weight of demand } d \\ c_{e} & \text { marginal cost of link } e \\ C & \text { assumed budget } \\ y_{e}=\frac{C}{\sum_{e} c_{e}} c_{e} & \text { capacity of link } e\end{array}$

\section{variables:}

$x_{d}$ flow allocated to demand $d$ maximize:

$$
F(x)=\sum_{d} w_{d} \log \left(x_{d}\right)
$$

constraints:

$$
\begin{aligned}
\sum_{d} a_{e d} x_{d} & \leq y_{e} \\
\text { for } \quad e & =1,2, \ldots, E \\
d & =1,2, \ldots, D
\end{aligned}
$$

In Task 3, between each O-D pair we choose the lowest cost path with respect to the links' marginal cost $c_{e}$ (lowest cost path routing). Note that the formulation allows for links of different capacities, but during the numerical evaluation we will consider equal sized links (where $c_{e} \equiv 1$ ). There is no closed formula available for the calculation of the allocations in this case, therefore we used the optimization tool "Solver" included in Microsoft Excel for numerical evaluations. We used the piece-wise approximation of the logarithmic revenue function as described in [5]. 


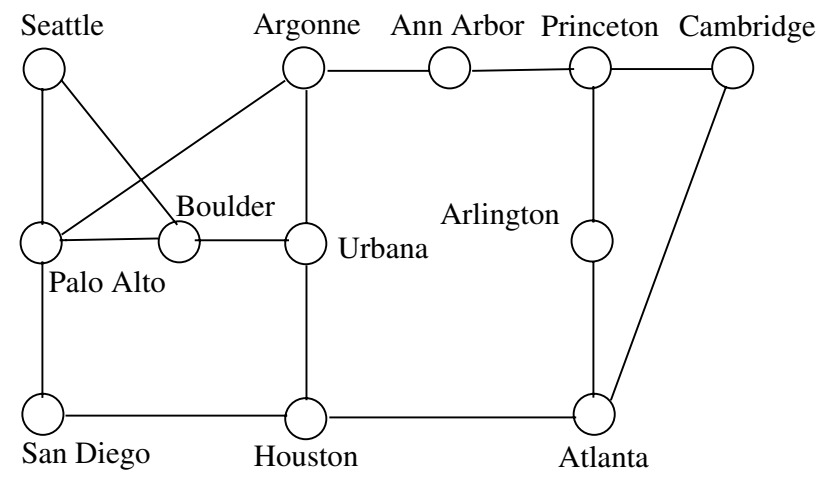

Fig. 1. The 12-node Example Network

\section{Numerical Examples}

\subsection{Input Parameters}

We consider the network of Figure 1. This network consists of 12 nodes and $E=16$ links. We assume that there is one demand between each network node pair yielding in total $D=66$ demands. We assume that each link has unit marginal cost (i.e. $c_{e} \equiv 1 \forall e$ ) and that each demand has equal weight $\left(w_{d} \equiv 10 \forall d\right)$.

\subsection{Numerical Results}

Recall from the formulation of Task 1 and Task 2 that the revenue is associated with the allocated bandwidth only, while the profit takes into account the link costs as well. Figure 2 compares the optimum revenue and profit as the function of the link cost budget $C$ of Task 1 ("Revenue" and "Profit") with those of Task 2 ("Max-Profit" and "Max-Revenue"). Recall that for a fixed budget $C$, Task 1 determines the bandwidth of each flow $\left(x_{d}\right)$ and the capacity of each link $\left(y_{e}\right)$ such that the revenue is maximized (see (1)). Since Task 1 uses the equality budget constraint, in this case the profit is maximized when the revenue is maximized and the difference between these two quantities is exactly $C$. (Indeed, see the "Revenue" and "Profit" curves.) For Task 2, $x_{d}, y_{e}$ and the actually used link budget are determined such that the profit is maximized (see (11)). (That is, for Task 2, the horizontal axis corresponds to the maximum allowable budget $C_{0}$ of (12).) Figure 2 plausibly highlights the difference between the revenue and profit maximization tasks. Increasing the budget up to a certain limit leads to the increase of both the "Profit" and "Max-Profit". Increasing the budget beyond this point (in this example at $C=660$ ) decreases the "Profit" of Task 1 and leads to the saturation of "Max-Profit" of Task 2, since in the profit maximization case the actually used budget may be smaller than the allowable. Task 2 effectively "freezes" the revenue increase that would lead to profit decrease (see the curve "Max-Revenue"). 


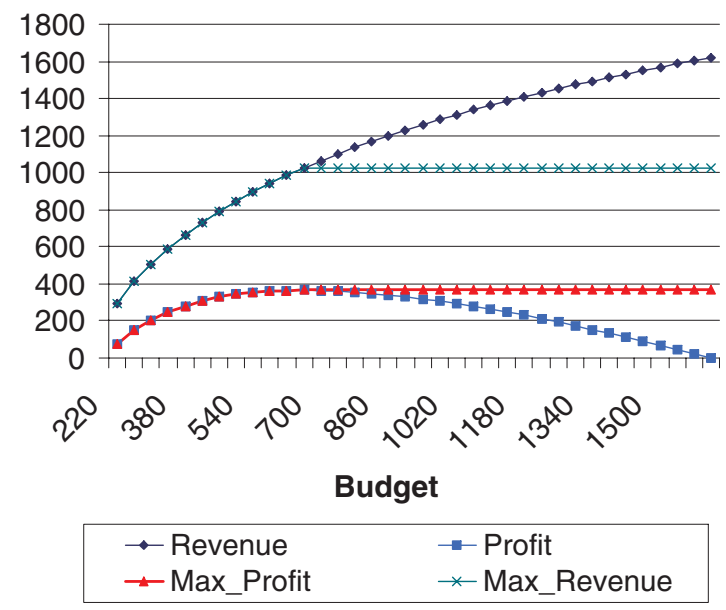

Fig. 2. Revenue and Profit for Task 1 and Task 3

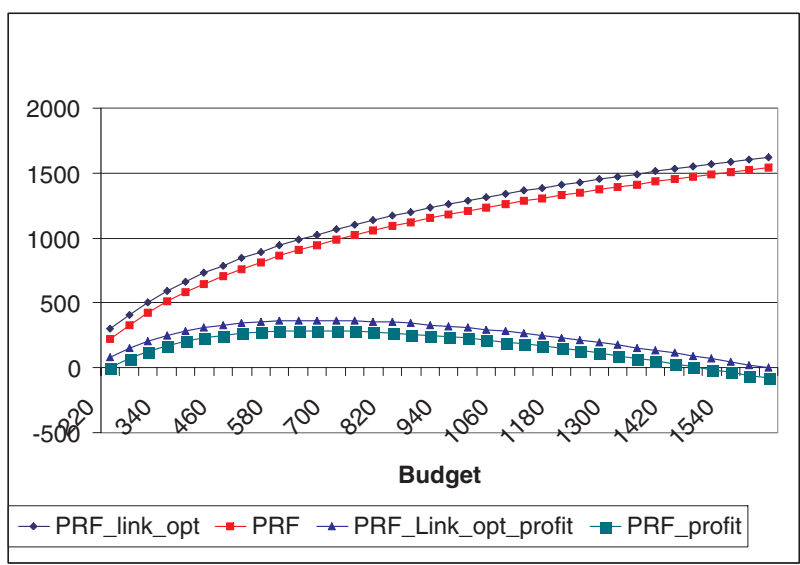

Fig. 3. Revenue and profit for fixed and optimized link PRF

In Figure 3 we compare the revenue and the profit as the function of the link cost budget $C$ of Task 1 ("PRF_link_opt" and"PRF_link_opt_profit") with those provided by the fixed link proportional fair method of Task 3 ("PRF" and "PRF_profit"). We can see again analogously to Figure 2 the logarithmic increase of the revenue in case of both methods and the saturation of the profit function around the same link cost budget value of $C=660$. Note that both the revenue and consequently the profit values are significantly higher when the link capacities are optimized (Task 1). Moreover, the difference between the two methods is approximately constant for both aspects.

The differences are even more visible in Figure 4 that highlights the difference between the revenue ("Revenue") and the profit ("Profit") values of Task 1 and Task 


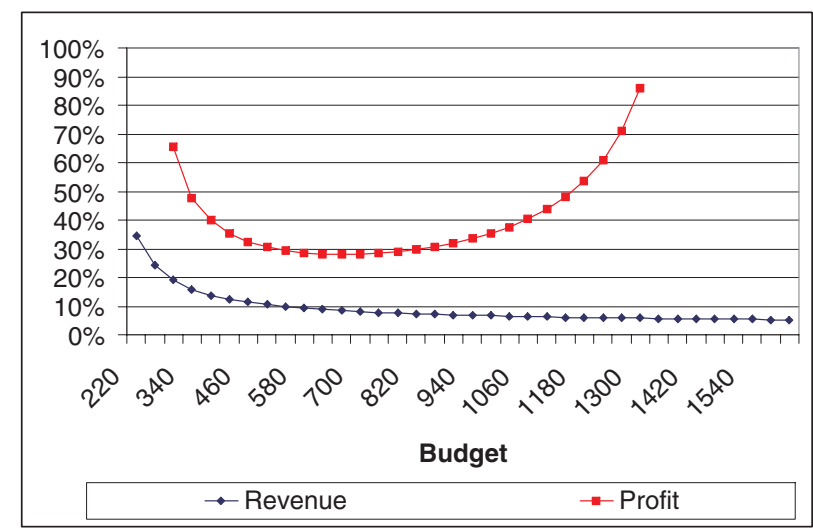

Fig. 4. Revenue and profit for fixed and optimized link PRF II.

3. The vertical axis is the "gain of Task 1 as compared to Task 3 ": it is calculated as the profit (and revenue) given by the optimized link proportional fair method (Task 1) divided by that of the fixed link case (Task 3), minus 1 .

In accordance with the approximately constant difference between the revenues provided by Task 1 ("PRF_link_opt") and Task 3 ("PRF") outlined in Figure 3, the relative gain provided by Task 1 is monotonously decreasing. We can observe in Figure 3. that at low capacity budget values, the profit of both Task 1 ("PRF_link_opt_profit") and Task 3 ("PRF_profit") are low due to the insufficient budget. Thus, the relative gain in Figure 4 when optimizing the capacity (Task 1) as compared to the fixed capacity case (Task 3 ) is high. As the capacity budget increases, the reachable optimum profit grows, and as the difference between the Task 1 and Task 3 is more or less constant, the relative profit gain decreases, arriving to its minimum at the optimal capacity budget. Note, that even in this case link capacity dimensioning adds (in our example approximately 30\%) to the profit as compared to the fixed capacity case. When the budget goes beyond the optimum, the profit decreases again and the relative gain of Task 1 as compared to Task 3 becomes higher again.

\section{Conclusion}

In this paper we considered link capacity dimensioning and bandwidth allocation in proportionally fair networks. We first considered the "basic task" (Task 1) where one is interested in finding the flow bandwidths and link capacities such that the revenue is maximized. For this case we provided explicit exact formulae. An important variant of this type of optimization problem arises when the cost associated with the total link capacities is not kept fixed but upper-bounded. In this case (Task 2) the profit (defined as the difference between revenue and cost) is maximized by using a portion of the total allowed link capacity budget. As a "reference case" for comparisons, we also considered the proportional fair sharing method with fixed link capacities (Task 3 ). 
We summarize our findings as follows:

- Under the fixed budget constraint, when both the paths for the flows realizing the demands and the link capacities are subject to optimization, the optimal solution assigns each flow to its shortest (with respect to the links' marginal cost) path.

- Under the inequality budget constraint, i.e. when the actually used link capacity budget can be chosen up to a bound, the maximum profit can be reached at a significantly lower capacity budget value than the maximum allowed budget.

- Link capacity dimensioning in proportionally fair networks may significantly increase the profit as compared to the case when the link capacities are fixed.

We believe that our problem formulations in Task 1 and Task 2 can provide important insight into traffic engineering problems and can serve as a basis for practically useful engineering tools. In future work we plan to present results for the case when the user flows are both lower- and upper bounded as the model in [17].

\section{References}

1. G. R. Ash, "Traffic Engineering \& QoS Methods for IP-, ATM-, \& TDM-Based Multiservice Networks", draft-ietf-tewg-qos-routing-00.txt, http://www.ietf.org/internet-drafts/draftietf-tewg-qos-routing-00.txt, Internet Engineering Task Force, work in progress, November 2000.

2. D. O. Awduche, A. Chiu, A. Elwalid, I. Widjaja, Xipeng Xiao, "A Framework for Internet Traffic Engineering" draft-ietf-tewg-framework-02.txt, http://www.ietf.org/internet-drafts/draftietf-tewg-framework-02.txt, Internet Engineering Task Force, work in progress, July 2000.

3. A. Fichou, C. Galand, S. Fdida, Y. Moret, "Evaluation of the ER Algorithm ERAQLES in Different ABR Environments", $5^{\text {th }}$ IFIP TC6 Workshop on Performance Modeling and Evaluation of ATM Networks, pp. 48/1-48/3, Ilkley, UK, July 1997.

4. G. Fodor, G. Malicsko, M. Pioro, "Link Capacity Dimensioning and Path Optimization for Networks Supporting elastic Services", submitted to ICC 2002, New York, USA, 2002.

5. G. Fodor, G. Malicsko, M. Pioro and T. Szymanski, "Path Optimization for Elastic Traffic under Fairness Constraints", $17^{\text {th }}$ International Teletraffic Congress, Salvador da Bahia, Brasil, 2001.

6. A. Goel, A. Meyerson, S. Plotkin, "Combining Fairness with Throughput: Online Routing with Multiple Objectives", To appear in JCSS, special issue on Internet Algorithms (Invited Paper) Extended abstract in STOC 2000

7. Y. T. Hou, H. H-Y. Tzeng, S. S. Panwar, "A Generic Weight-Based Network Bandwidth Sharing Policy for ATM ABR Service", IEEE International Conference on Communications, ICC '98, pp. 1492-1499, 1998.

8. K. Kar, M. Kodialam, T. V. Lakshman, "Minimum Interference Routing of Bandwidth Guaranteed Tunnels with MPLS Traffic Engineering Applications", IEEE Journal on Selected Areas in Communications, Vol. 18, No. 12, pp. 2566-2579, Dec. 2000.

9. F. P. Kelly, A. K. Mauloo, D. K. H. Tan, "Rate Control for Communication Networks: Shadow Prices, Proportional Fairness and Stability", Journal of the Operational Research Society, (49), pp. 206-217, August, 1997.

10. F. P. Kelly, A. K. Maulloo, D. K. H. Tan, "Rate Control for Communication Networks: Shadow Prices, Proportional Fairness and Stability", Journal of the Operational Research Society, (49), pp. 237-252, 1998. 
11. J. Kleinberg, "Single-Source Unsplittable Flow", IEEE Symposium on Foundations of Computer Science, 1996.

12. J. Kleinberg, Y. Rabani, É. Tardos, "Fairness in Routing and Load Balancing", IEEE Symposium on Foundations of Computer Science, 1999.

13. J. Lawrence, "Designing Multiprotocol Label Switching Networks", IEEE Communication Magazine, pp. 134-142, Vol. 39, No. 7, July 2001.

14. Q. Ma, P. Steenkiste, H. Zhang, "Routing High-bandwidth Traffic in Max-min Fair Share Networks", SIGCOMM '97, pp. 206-217, August, 1997.

15. L. Massoulie, J. W. Roberts, "Bandwidth Sharing and Admission Control for Elastic Traffic", International Teletraffic Specialist Seminar, Yokohama, 1998.

16. L. Massoulie, J. W. Roberts, "Bandwidth Sharing: Objectives and Algorithms", IEEE INFOCOM '99, 1999.

17. M. Pioro, "On Some Dimensioning Tasks Associated with the Notion of Proportional Fairness", Technical Report, Lund Institute of Technology at Lund University, CODEN:LUTEDX(TETS-7181)/1-6/(2001)\&local 18, October 2001. http://www.telecom.lth.se/Personal/Michal.Pioro/report.pdf

18. S. Plotkin, "Competitive Routing of Virtual Circuits in ATM Networks", IEEE Journal of Selected Areas in Communications, Vol. 13, No. 6, pp.1128-36, Aug. 1995.

19. H. Qingyanga, D.W. Petr, "Global Max-Min Fairness Guarante for ABR Flow Control", IEEE INFOCOM' '98, 1998.

20. B. Teitelbaum, S. Hares, L. Dunn, R. Neilson, V. Narayan, F. Reichmeyer, "Internet2 QBone: Building a Testbed for Differentiated Services", IEEE Network, Vol. 13, No. 5, pp. 8-16, September/October 1999.

21. W. K. Tsai, M. Iyer, "Constraint Precedence in Max-Min Fair Rate Allocation", IEEE International Conference on Communications, ICC, 2000.

22. G. de Veciana, T-J. Lee, T. Konstantopoulos, "Stability and Performance Analysis of Networks Supporting Elastic Services", IEEE/ACM Transactions on Networking, Vol. 9, No. 1, pp. 2-14, Feb. 2001.

23. Andreu Mas-Collel, Michael D. Whinston, Jerry R. Green, "Microeconomic Theory" New York, Oxford University Press 1995. 